\title{
Avaliação da seleção brasileira feminina de basquete
}

\author{
D.A.S. Gentil ${ }^{1}$, C.P.S. Oliveira ${ }^{2}$, T.L. Barros Neto ${ }^{3}$ e V.L. Tambeiro ${ }^{4}$
}

\section{RESUMO}

Foram avaliadas 13 jogadoras de basquete convocadas para disputa dos Jogos Olímpicos em Sidney - 2000. As atletas foram submetidas à mensuração do peso e altura, a um teste ergoespirométrico em esteira e a um teste de impulsão vertical. Todos os testes foram realizados no Cemafe (Centro de Medicina da Atividade Física e do Esporte) da Unifesp/EPM, em junho de 2000. Os resultados encontrados estão descritos a seguir: idade média de $24,4 \pm 4,6$ anos, peso corporal 70,8 $\pm 8,6 \mathrm{~kg}$, estatura $182 \pm 7,6 \mathrm{~cm}$, e índice de massa corpórea (IMC) $21,4 \pm 1,8$. A avaliação ergoespirométrica revelou valores para o consumo máximo de oxigênio (V̇O2 máx) de 49,9 $\pm 5,4 \mathrm{ml} / \mathrm{kg} / \mathrm{min}$; o consumo de oxigênio no limiar anaeróbio (VंO2 LA) de 38,7 \pm 4,3 $\mathrm{ml} / \mathrm{kg} / \mathrm{min}$; freqüência cardíaca máxima (FC máx) 194,4 $\pm 9,6 \mathrm{bpm}$; freqüência cardíaca no limiar anaeróbio (FC LA) 179,7 \pm 5,7bpm; velocidade no limiar anaeróbio (VEL LA) $11,9 \pm 1,4 \mathrm{~km} / \mathrm{h}$; gasto calórico no limiar anaeróbio (GASTO CAL) $815,6 \pm 71,8 \mathrm{kcal} / \mathrm{h}$. No teste de impulsão vertical as atletas foram orientadas a saltar com a maior potência e o maior número de vezes possível em 30 segundos, detectando-se os índices por equipamento sonar (Vertisonic ${ }^{\circledR}$ ). Foram encontrados para a potência máxima ou pico de potência $9,9 \pm 2,2 \mathrm{~W}$ e um trabalho muscular total durante os 30 segundos de $602,1 \pm 104,8 \mathrm{~J}$. A avaliação médica e fisiológica é fundamental para atletas de alto nível alcan-

1. Médico do Esporte; Fisiologista da Seleção Brasileira Feminina de Basquete; Mestre em Reabilitação - Unifesp/EPM.

2. Médico do Esporte; Chefe do Departamento Médico da Confederação Brasileira de Basquete; Docente das Disciplinas Fundamentos Biológicos e Cinesiologia do Curso de Educação Física do Núcleo da Saúde da UNIFMU.

3. Coordenador do Cemafe; Docente do Departamento de Fisiologia da Unifesp/EPM.

4. Secretária Executiva do Cemafe.

Recebido em: 17/10/2000.

Aceito em: 21/2/2001. çarem sucesso em competições de nível internacional, sendo estes parâmetros essenciais ao planejamento e periodização do treinamento.

Palavras-chave: Fisiologia do exercício. Basquete feminino. Avaliação física.

\section{ABSTRACT}

\section{Evaluation of Brazilian female basketball team}

Thirteen (13) elite female basketball players were submitted to medical and physiological evaluation in Cemafe (Federal University of São Paulo) in June, 2000. Age was $24.4 \pm 4.6$ y, height $182 \pm 7.6 \mathrm{~cm}$, and body mass $70.8 \pm$ $8.6 \mathrm{~kg}$. The Brazilian team was submitted to an ergospirometry evaluation by a computerized analysis of expired gases with cardiovascular responses recorded by ECG. A vertical jump test was performed to detect the anaerobic power during 30 seconds. The results were: maximum oxygen uptake ( $\dot{\mathrm{V} O 2} \mathrm{max}$ ) $49.9 \pm 5.4 \mathrm{ml} / \mathrm{kg} / \mathrm{min}$; threshold oxygen uptake ( $\dot{V} O 2 \mathrm{LA}$ ) $38.7 \pm 4.3 \mathrm{ml} / \mathrm{kg} / \mathrm{min}$; maximum heart rate $194.4 \pm 9.6$ bpm; anaerobic threshold heart rate $179.7 \pm$ $5.7 \mathrm{bpm}$; anaerobic threshold velocity $11.9 \pm 1.4 \mathrm{~km} / \mathrm{h}$; anaerobic power (peak) $9.9 \pm 2.2 \mathrm{~W}$; total muscular work (30 s) $602.1 \pm 104.8 \mathrm{~J}$. Medical and physiological evaluation is compulsory for elite female basketball players to be successful in international championships.

Key words: Physiology of exercise. Female basketball. Evaluation.

\section{INTRODUÇÃO}

O basquete teve sua primeira partida oficial disputada em 1892 em Springfield, nos Estados Unidos; atualmente são cerca de 300 milhões de praticantes em todo mundo. Isto mostra uma popularidade impressionante, que movimenta bilhões de dólares. Com esse incrível número de participantes e maiores premiações, as competições se tornaram mais acirradas e a partir disso a preparação física se tornou imprescindível para a vitória, abrindo grande espaço para a fisiologia de exercício. 
É muito difícil definir com precisão quais são as exigências fisiológicas durante uma partida competitiva de basquete $^{1}$. O número de estudos científicos que fazem referência ao basquete ainda é escasso e a comparação entre eles é difícil, pois estes estudos, em sua grande maioria, são diferentes (objetivo, nível de treinamento, estilo de jogo ou idade $)^{1}$.

Pelas próprias regras e características do jogo, observase, durante uma partida, rápidas transições entre ataque $\mathrm{e}$ defesa, a fluência de movimentos e múltiplas responsabilidades para todos os jogadores (arremessos, rebotes, ataque, defesa e contra-ataque, por exemplo). A maior parte dos estudos apenas descreve as características fisiológicas de jogadores ou times, de diferentes níveis.

Apenas McInnes ${ }^{2}$ em 1995, observando jogadores de alto nível, tentou caracterizar os padrões de movimento durante um jogo competitivo. McInnes et al. dividiram as ações dentro de uma partida de basquete em oito categorias (andar/em pé; trotar; correr; "tiros"; deslocamentos pequenos; médios; grandes e saltar). Os resultados nos mostram a natureza intermitente do esporte, $997 \pm 183$ mudanças de posição durante o jogo (uma mudança de posição a cada 2 segundos, visto que em média o tempo de jogo é de 36,3 minutos por partida). Os resultados publicados nos mostram que os deslocamentos (em todas as direções) representaram 34,6\% dos movimentos de um jogo, 31,2\% das ações foram classificadas em corrida (do trote aos "tiros"). Os saltos representaram 4,6\% das ações e o ato de estar em pé ou andando, 29,6\%. Os movimentos considerados de alta intensidade eram repetidos a cada 21 segundos, perfazendo $15 \%$ do tempo do jogo.

Este estudo tem o objetivo de descrever as características (físicas e fisiológicas) da seleção feminina de basquete que representou o Brasil nos Jogos Olímpicos em Sidney 2000.

\section{MATERIAL E MÉTODO}

Foram avaliadas 13 jogadoras convocadas para início da preparação visando os Jogos Olímpicos em Sidney - 2000. Cabe ressaltar que quatro destas jogadoras avaliadas foram posteriormente "cortadas" da seleção por critérios exclusivamente técnicos.

Todas as avaliações foram realizadas no Cemafe (Centro da Medicina da Atividade Física e do Esporte) da Universidade Federal de São Paulo - Escola Paulista de Medicina (Unifesp/EPM), no mês de junho, em dois períodos (manhã e tarde) de dias consecutivos.

$\mathrm{O}$ peso e a altura das atletas foram mensurados em balança aferida sem a utilização de calçados.
A avaliação ergoespirométrica, em esteira (Lifestride modelo 7500), consistiu no registro de um eletrocardiograma em repouso (eletrocardiógrafo Dixtal $^{\circledR}$ ) e o registro de três derivações (MC5, v2, AVF) por todo teste. Aferiu-se a pressão arterial antes, no exercício máximo e durante a recuperação (segundo e quarto minutos). O protocolo estabelecido foi dois minutos em repouso, início do teste a $5 \mathrm{~km} / \mathrm{h}$ (por dois minutos) e a partir de daí, a cada minuto, a velocidade era aumentada em $1 \mathrm{~km} / \mathrm{h}(6 \mathrm{~km} / \mathrm{h}, 7 \mathrm{~km} / \mathrm{h}, 8 \mathrm{~km} /$ $h, .$.$) até a exaustão. As variáveis foram obtidas de forma$ direta (com máscara) pelo analisador de gases MiniVista CPX e o Software Vacumed turbo 4.0.

A avaliação da potência anaeróbia foi realizada por meio de teste da impulsão vertical, utilizando-se fixo no teto um aparelho sonar (Vertisonic ${ }^{\circledR}$ ), que mede a diferença de deslocamento. As atletas foram orientadas a realizar o maior número de saltos, na maior potência (o mais alto) possível, sem o auxílio dos membros superiores, por 30 segundos. $\mathrm{O}$ valor do deslocamento vertical era registrado um a um. A partir dos registros dos saltos, foram calculados a potência máxima (pico de potência) e trabalho muscular total realizado durante os 30 segundos, da seguinte maneira: o maior valor absoluto do salto, multiplicado pelo peso corporal expressa a potência máxima (pico de potência); a somatória de todos os saltos realizados, multiplicado pelo peso corporal, expressa o trabalho muscular total realizado durante os 30 segundos.

\section{RESULTADOS}

TABELA 1

Características físicas e posição de jogo da Seleção

Feminina Basquete Brasileira em preparação para os J ogos Olímpicos em Sidney - 2000

\begin{tabular}{clcccc}
\hline $\mathbf{N}$ & Posição & $\begin{array}{c}\text { Idade } \\
\text { (anos) }\end{array}$ & $\begin{array}{c}\text { Estatura } \\
\text { (cm) }\end{array}$ & $\begin{array}{c}\text { Peso } \\
\text { (kg) }\end{array}$ & IMC \\
1 & pivô & 36 & 190 & 71 & 19,7 \\
2 & ala & 29 & 179 & 61 & 19,0 \\
3 & ala & 25 & 173 & 64 & 21,4 \\
4 & ala & 28 & 174 & 68 & 22,5 \\
5 & armadora & 27 & 175 & 62 & 20,2 \\
6 & pivô & 20 & 185 & 79 & 23,0 \\
7 & ala & 21 & 180 & 66 & 20,0 \\
8 & pivô & 22 & 188 & 79 & 22,4 \\
9 & ala & 21 & 183 & 67 & 19,9 \\
10 & armadora & 21 & 168 & 65 & 23,0 \\
11 & pivô & 22 & 190 & 68 & 18,7 \\
12 & pivô & 20 & 192 & 88 & 23,8 \\
13 & pivô & 25 & 187 & 83 & 23,7 \\
\hline Média & & 24,4 & 182,0 & 70,8 & 21,4 \\
DP & & & $\pm 7,6$ & $\pm 8,6$ & $\pm 1,8$ \\
\hline
\end{tabular}


TABELA 2

Características fisiológicas da Seleção Olímpica de Basquete Feminina Brasileira em preparação para os J ogos Olímpicos em Sidney - 2000

\begin{tabular}{|c|c|c|c|c|c|}
\hline $\mathbf{N}$ & Posição & 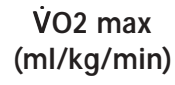 & $\begin{array}{c}\dot{\text { VO2 LA }} \\
\text { (ml/ kg/min) }\end{array}$ & $\begin{array}{l}\text { Vel LA } \\
(\mathbf{k m} / \mathbf{h})\end{array}$ & $\begin{array}{l}\text { Gasto Cal. } \\
\text { LA (kcal/h) }\end{array}$ \\
\hline 1 & pivô & 47,2 & 38,4 & 12 & 820 \\
\hline 2 & ala & 55,7 & 40,4 & 10 & 740 \\
\hline 3 & ala & 54,5 & 41,7 & 12 & 802 \\
\hline 4 & ala & 56,6 & 44,1 & 13 & 901 \\
\hline 5 & armadora & 55,3 & 45,1 & 13 & 849 \\
\hline 6 & pivô & 41,7 & 34,4 & 12 & 904 \\
\hline 7 & ala & 47,1 & 38,0 & 12 & 753 \\
\hline 8 & pivô & 41,6 & 32,3 & 10 & 785 \\
\hline 9 & ala & 53,4 & 42,7 & 14 & 846 \\
\hline 10 & armadora & 50,8 & 37,1 & 14 & 723 \\
\hline 11 & pivô & 51,8 & 40,4 & 12 & 825 \\
\hline 12 & pivô & 50,6 & 37,4 & 11 & 987 \\
\hline 13 & pivô & 42,2 & 31,0 & 10 & 773 \\
\hline Média & & 49,9 & 38,7 & 11,9 & 815,6 \\
\hline DP & & $\pm 5,4$ & $\pm 4,3$ & $\pm 1,4$ & $\pm 71,8$ \\
\hline
\end{tabular}

TABELA 3

Características da potência anaeróbia aláctica da Seleção Olímpica de Basquete Feminina Brasileira em preparação para os J ogos Olímpicos em Sidney - 2000

\begin{tabular}{clcc}
\hline $\mathbf{N}$ & Posição & $\begin{array}{c}\text { Potência máxima } \\
(\mathbf{W})\end{array}$ & $\begin{array}{c}\text { Trabalho total } \\
\mathbf{(})\end{array}$ \\
1 & pivô & 9,2 & 509 \\
2 & ala & - & - \\
3 & ala & 7,0 & 480 \\
4 & ala & 8,9 & 613 \\
5 & armadora & 6,2 & 522 \\
6 & pivô & 13,1 & 732 \\
7 & ala & 11,6 & 535 \\
8 & pivô & 12,5 & 740 \\
9 & ala & 8,2 & 530 \\
10 & armadora & 8,6 & 800 \\
11 & pivô & 12,1 & 612 \\
12 & pivô & 11,0 & 624 \\
13 & pivô & 10,6 & 528 \\
\hline Média & & 9,9 & 602,1 \\
DP & & $\pm 2,2$ & $\pm 104,8$ \\
\hline
\end{tabular}

A atleta 2 não realizou o teste de impulsão vertical por recomendação médica.

\section{DISCUSSÃO}

Os mais recentes estudos relacionados ao basquete (principalmente universitário) dão importância muito maior à estatura e ao metabolismo anaeróbio (potência anaeróbia) que a outras características físicas e fisiológicas ${ }^{3-7}$. Estu- dos de fisiologia do exercício em equipes de alto nível (seleções nacionais e NBA) confirmam o metabolismo anaeróbio (potência anaeróbia) como a característica mais importante, mas associam a potência aeróbia (consumo máximo de oxigênio e consumo de oxigênio no limiar anaeróbio), agilidade e velocidade ao sucesso nas competições mais importantes ${ }^{8,9}$.

A importância da potência aeróbia estaria mais relacionada aos processos de recuperação (tanto da freqüência cardíaca, quanto à remoção de lactato sanguíneo) do que à performance propriamente dita ${ }^{10,11}$. Durante uma partida de basquete (pelas regras utilizadas atualmente) ocorrem diversas paralisações (tempos solicitados pelo técnico, tempos para a cobertura da televisão, arremessos de lances livres, entre outras) o que permite que o atleta tenha pequenos períodos de recuperação durante o jogo.

Os resultados por nós obtidos, em grande parte, vão ao encontro da literatura pesquisada.

A média do peso corporal da seleção feminina brasileira foi de $70,8 \pm 8,6 \mathrm{~kg}$, sendo este valor acima do descrito por outros autores $(61,5-68,3 \mathrm{~kg})^{6,8,9,12,13}$. Cabe lembrar que alguns destes estudos avaliaram jogadoras universitárias. As armadoras foram as jogadoras mais leves $(63,5 \mathrm{~kg})$, em relação às alas $(65,2 \mathrm{~kg})$ e às pivôs $(78 \mathrm{~kg})$. Smith e Thomas ${ }^{9}$ encontraram a mesma relação para as posições de jogo, sendo que as armadoras $(67,3 \mathrm{~kg})$ foram significantemente mais leves que as alas $(77,1 \mathrm{~kg})$ e que as pivôs $(81,1 \mathrm{~kg})$.

A maior estatura, o maior peso corporal e um maior percentual de gordura, das jogadoras que atuam como pivô, estão diretamente relacionados às características da posição, que exige um maior contato físico, principalmente a disputa pelo rebote dentro do garrafão.

O comportamento da pressão arterial, da frequiência cardíaca e todos os traçados eletrocardiográficos (antes, durante e após o teste) foram considerados dentro dos padrões de normalidade para atletas de alto nível.

A média potência aeróbia, expressa pelo consumo máximo de oxigênio (VंO2 máx), encontrada foi 49,9 $\pm 5,4 \mathrm{ml} /$ $\mathrm{kg} / \mathrm{min}$, que está próxima aos maiores valores descritos, que estiveram entre 36 e $51 \mathrm{ml} / \mathrm{kg} / \mathrm{min}^{8,9,12-15}$. O consumo de oxigênio no limiar anaeróbio ( $\mathrm{VO} 2 \mathrm{LA}$ ) registrado em nosso estudo foi de $38,7 \pm 4,3 \mathrm{ml} / \mathrm{kg} / \mathrm{min}$, não sendo encontrado nenhum valor para comparação. Os valores encontrados para armadoras e alas foram maiores que para as pivôs.

Como já foi citada, a potência aeróbia para jogadoras de basquete é uma variável imprescindível ao bom desempenho em jogos de alto nível, sendo muito importante na recuperação dentro da própria partida.

A potência anaeróbia assume importância no bom desempenho em qualquer nível de jogo. Atualmente não existe 
uma uniformização para sua avaliação; tem sido determinada de diversas maneiras, entre elas, o teste de força de Margaria-Kalman ${ }^{16}$, impulsão vertical utilizando a Fórmula de Lewis ${ }^{6,17}$ ou Harmon ${ }^{15,18}$, impulsão vertical sobre uma plataforma de força ${ }^{14,19}$ e o teste de Wingate ${ }^{10,14}$.

Em nosso estudo, optamos pela impulsão vertical medida pelo aparelho sonar (Vertisonic $\left.{ }^{\circledR}\right)$, por considerarmos o movimento mais específico para o basquete. Encontramos uma média para a potência máxima (pico de potência) de $9,9 \pm 2,2 \mathrm{~W}$ e para o trabalho muscular total nos 30 segundos de $602,1 \pm 104,8 \mathrm{~J}$, sendo a potência máxima (pico de

\section{REFERÊNCIAS}

1. Hoffman JR, Maresh CM. Exercise and sport science. $1^{\text {st }}$ ed. Philadelphia: Lippincott Williams \& Wilkins, 2000;46:733-44.

2. McInnes SE, Carlson JS, Jones CJ, McKenna MJ. The physiological load on basketball players during competition. J Sport Sci 1995;13:38797.

3. Chandler J. Goals and activities for athletic conditioning for basketball. NSCA J 1986;8:52-5.

4. Fox E. Sports physiology. $2^{\text {nd }}$ ed. Philadelphia: WB Saunders.

5. Gillam GM. Physiological basis of basketball bioenergetics. NSCA J 1985;6:44-71.

6. Gillam GM. Identification of anthropometric and physiological characteristics relative to participation in college basketball. NSCA J 1985;7: 34-6.

7. Hoffman JR, Tennenbaum G, Maresh CM, Kraemer WJ. Relationship between athletic performance tests and playing in elite college basketball players. J Strength Cond Res 1996;10:67-71.

8. McArdle WD, Magel JR, Kyvallos LC. Aerobic capacity, heart rate and estimated energy cost during women's competitive basketball. Res Q 1971:42:178-86.

9. Smith HK, Thomas SG. Physiological characteristics of elite female basketball players. Can J Sport Sci 1991;16:289-95.

10. Hoffman JR, Epstein S, Einbinder M, Weinstein I. The influence of aerobic capacity on recovery from high intensity exercises in basketball players. J Strength Cond Res 1999 (in press). potência) e o trabalho muscular total maior nas pivôs em relação às armadoras e alas.

A avaliação médica e fisiológica é fundamental para atletas de alto nível alcançarem sucesso em competições de nível internacional, sendo estes parâmetros essenciais ao planejamento e periodização do treinamento.

Os resultados por nós encontrados na avaliação da Seleção Brasileira de Basquete Feminina vão, em grande parte, ao encontro do que está descrito em trabalhos publicados em revistas internacionais indexadas sobre o mesmo tema.

11. Hoffman JR. The relationship between aerobic fitness and recovery from high-intensity exercise in infantry soldiers. Mil Med 1997;162:484-8.

12. Vaccaro P, Clarke DH, Wrenn JP. Physiological profiles of elite women basketball players. J Sports Med 1979;19:45-54.

13. Morrow JR, Hosler WW, Nelson JK. A comparison of women intercollegiate basketball players, volleyball and non-athletes. J Sports Med 1980; 20:435-40.

14. Hakkinen K. Changes in physical fitness profile in female basketball players during the competitive season including explosive type strength training. J Sports Med Phys Fitness 1993;33:19-26.

15. Petko M, Hunter GR. Four-year changes in strength, power and aerobic fitness in women college basketball players. Strength Cond 1997;19: 46-9

16. Coleman AE, Kreuzer P, Friedrich DW, Juvenal JP. Aerobic and anaerobic responses of male college freshmen during a season of basketball. J Sports Med 1974;14:26-31.

17. Latin RW, Berg K, Baechle T. Physical and performance characteristics of NCAA division I male basketball players. J Strength Cond Res 1994; 8:214-8.

18. Hunter GR, Hilyer J, Foster MA. Changes in fitness during 4 years of intercollegiate basketball. J Strength Cond Res 1993;7:26-9.

19. Hoffman JR, Stavsky H, Falk B. The effect of water restriction on anaerobic power and vertical jumping height in basketball players. Int J Sports Med 1995;16:214-8. 